\title{
Subarachnoid Hemorrhage in the Presence of Both Intracranial Dissecting and Saccular Aneurysms
}

\author{
-Two Case Reports- \\ Yasuhiko AKIYAMA, Kouzo MORITAKE, Takeshi MIYAZAKI, \\ and Hidemasa NAGAI
}

Department of Neurosurgery, Shimane University School of Medicine, Izumo, Shimane

\begin{abstract}
Two patients presented with subarachnoid hemorrhage (SAH) associated with both intracranial dissecting and saccular aneurysms. Case 1, a 48-year-old woman, had a saccular aneurysm of the right internal carotid artery and dissecting aneurysms of the bilateral vertebral arteries. Case 2, a 52-year-old man, had three saccular aneurysms in the anterior circulation and a dissecting aneurysm of the unilateral vertebral artery. A saccular aneurysm was responsible for the SAH in both patients. Ruptured saccular aneurysms were treated with surgical clipping and unruptured dissecting aneurysms remained untreated. SAH recurred due to bleeding from an untreated dissecting aneurysm 4 days after the initial SAH in Case 1. Triple-H therapy, which causes increased hemodynamic stress, was not administered for symptomatic cerebral vasospasm after SAH in Case 2, because of the risk of bleeding from the untreated dissecting aneurysm, and the patient suffered cerebral infarction. The risk factors for this rare association are unclear, but both patients were smokers and had hypocholesterolemia including low apolipoprotein E levels. The clinical management of patients with SAH and both dissection and saccular aneurysms is complicated. Asymptomatic dissecting aneurysm has a benign clinical course in general, but hemodynamic stress related to stroke may induce abrupt development of dissecting aneurysms. Prophylactic obliteration during the acute stage of $\mathrm{SAH}$ may provide better outcomes if the unruptured dissecting lesion appears as obvious aneurysmal dilatation or pearl-andstring sign and is safely treatable with endovascular trapping.
\end{abstract}

Key words: multiple cerebral aneurysms, intracranial dissecting aneurysm, saccular aneurysm, subarachnoid hemorrhage, clinical management

\section{Introduction}

Intracranial dissecting aneurysms carry high risk for subarachnoid hemorrhage (SAH) and cerebral ischemia, and the reported incidence has been increasing. ${ }^{1,4-7,9,10,14,21)}$ Multiple cerebral aneurysms, even of the same type, complicate the therapeutic management in the presence of SAH. Preoperative identification of the ruptured lesion, and decisions regarding the management of the ruptured aneurysms and coexisting unruptured aneurysms can be difficult. Consensus has been reached on the surgical strategies to approach multiple saccular aneurysms, ${ }^{3)}$ but the optimal clinical management of simultaneous intracranial dissecting and saccular aneurysms remains to be determined.

Received May 15, 2006; Accepted October 23, 2006
We treated two patients with SAH in the presence of both dissecting and saccular cerebral aneurysms.

\section{Case Reports}

Case 1: A 48-year-old woman suddenly suffered severe headache, vomiting, and subsequent loss of consciousness. She had a history of smoking 20 cigarettes per day since age 20 years. On admission, she had Glasgow Coma Scale (GCS) score 8. Computed tomography (CT) revealed diffuse $\mathrm{SAH}$, but the hematoma appeared to be most prominent in the right sylvian cistern and quadrigeminal cistern (Fig. 1). No hematoma was found in the cisterns in the posterior cranial fossa, fourth ventricle, cerebellar convexity, or surroundings of the brain stem. Angiography revealed a small saccular aneurysm of the right internal carotid artery and dilatations of 


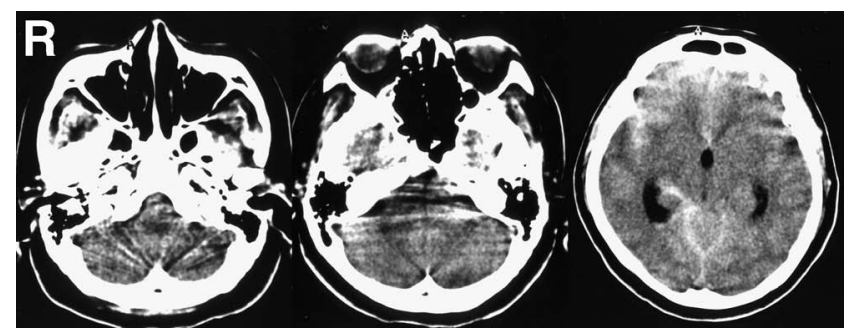

Fig. 1 Case 1. Computed tomography scans on admission demonstrating a subarachnoid hematoma in the interhemispheric, right sylvian, and right ambient cisterns. The subarachnoid hemorrhage is thick in the right hemisphere, but not observed in the cerebellar convexity or surroundings of the brain stem.
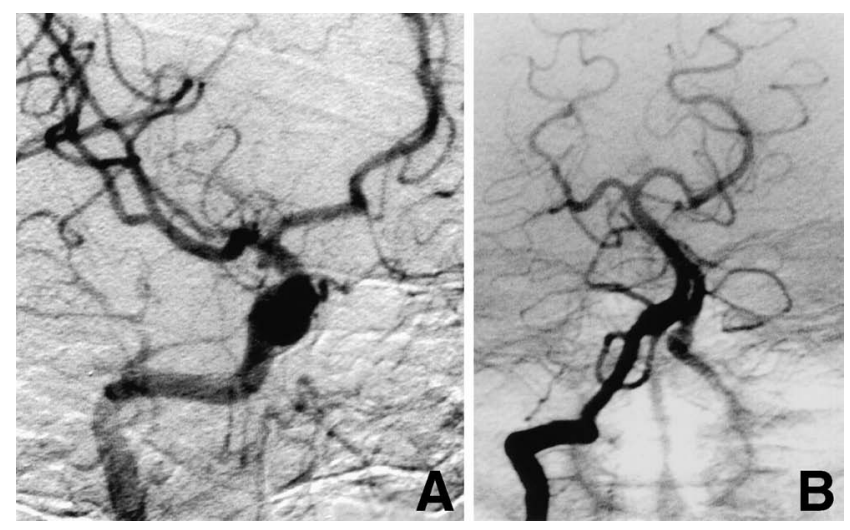

Fig. 2 Case 1. A: Right carotid angiogram, right anterior oblique view, showing an aneurysm of the internal carotid artery. B: Right vertebral angiogram, anteroposterior view, demonstrating aneurysmal dilatations of the bilateral vertebral arteries.

the bilateral intracranial vertebral arteries, suggesting the presence of dissecting aneurysms (Fig. 2). We concluded that the SAH originated from rupture of the aneurysm of the right internal carotid artery.

Clipping surgery was performed via the right pterional approach on the day of SAH onset. Thick subarachnoid hematoma was found in the right sylvian cistern and around the right internal carotid artery. Clot adhesion on the aneurysm confirmed that rupture of the aneurysm of the right internal carotid artery was responsible for the SAH. Fenestration of Liliequist's membrane showed that the prepontine cistern and basilar artery trunk were free of the thick subarachnoid hematoma. Cisternal drainage was placed. Surgical procedures were concluded uneventfully and her GCS score im-

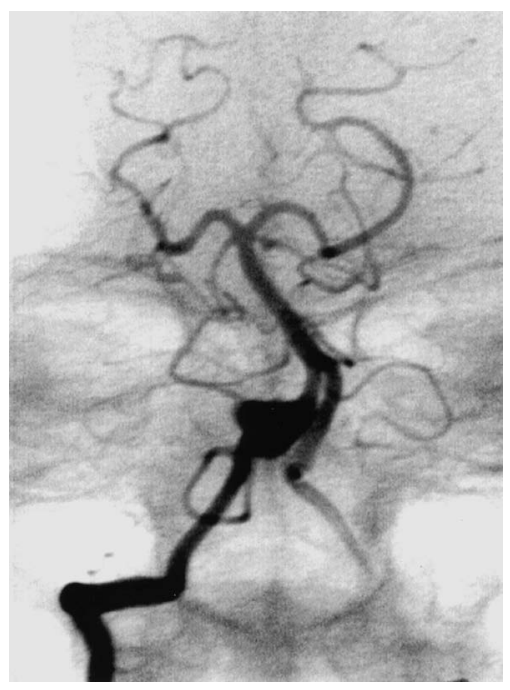

Fig. 3 Case 1. Right vertebral angiogram revealing an enlarged dissecting aneurysm.

proved to 13 on the next day.

Her systolic blood pressure was controlled below $140 \mathrm{mmHg}$ after the surgery because the dissecting aneurysms remained untreated. However, she complained of another sudden headache on the 4th postoperative day. Approximately $550 \mathrm{ml}$ of fresh blood flowed out of the cisternal drainage. Emergency CT demonstrated SAH in the basal cistern and hematomas in the third and fourth ventricles. Fortunately, there was no massive intracranial hematoma since most of the hemorrhage had escaped via the cisternal drainage. Conventional angiography revealed enlargement of the dissecting aneurysm of the right vertebral artery, suggesting new bleeding from this site (Fig. 3). She underwent endovascular treatment, and was discharged without neurological sequelae. The dissecting aneurysm of the left vertebral artery was closely monitored by conventional angiography, which showed improvement at the 1-year follow-up examination.

Case 2: A 52-year-old man suddenly groaned while asleep and then became restless and confused. CT revealed a thick hematoma in the basal, ambient, and bilateral sylvian cisterns (Fig. 4). Angiography revealed saccular aneurysms of the bilateral middle cerebral arteries and the right internal carotid artery, and a dissecting aneurysm of the left vertebral artery (Fig. 5). He had no relevant medical history, but had smoked 30-40 cigarettes a day since age 16 years. As the largest saccular aneurysm was located on the left middle cerebral artery and the SAH was thickest in the left sylvian cistern, we concluded that the bleeding was due to rupture of 
the left middle cerebral artery aneurysm.

Emergency surgical clipping was performed. He developed right hemiparesis starting on the 8th postoperative day, which we regarded as the clinical manifestation of cerebral vasospasm after SAH. However, we hesitated to administer triple- $\mathrm{H}$ therapy ${ }^{18)}$ because of the risk of bleeding from the untreated dissecting aneurysm due to increased hemodynamic stress. He suffered cerebral infarc-

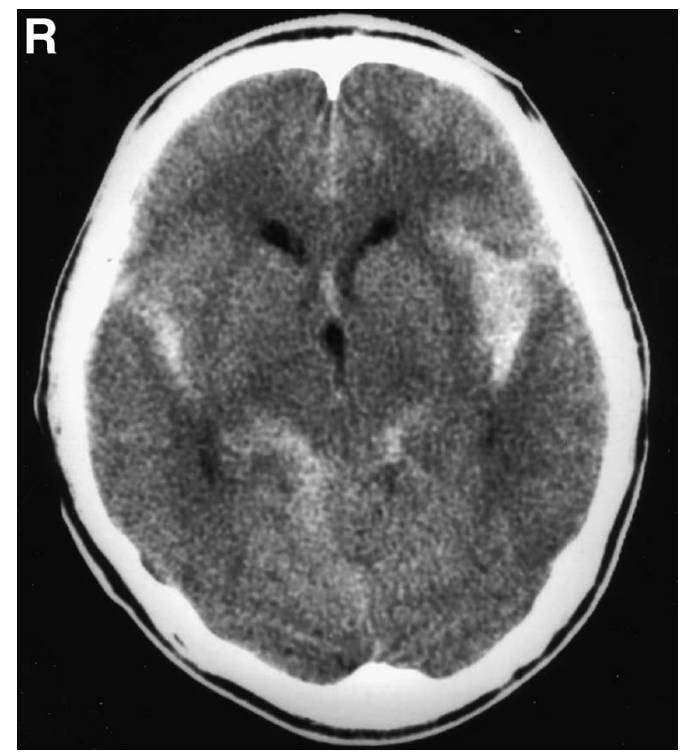

Fig. 4 Case 2. Computed tomography scan on admission demonstrating a subarachnoid hematoma in the interhemispheric cistern and bilateral sylvian and ambient cisterns, and thickest in the left sylvian cistern. tion on day 10 after SAH. The remaining saccular aneurysms of the right middle cerebral artery and the right internal carotid artery were clipped 4 weeks later. As the dissecting aneurysm of the left vertebral artery remained asymptomatic throughout with lower control of blood pressure, we decided to continue careful monitoring. He was discharged with right hemianopsia and mild right hemiparesis attributable to cerebral vasospasm.

\section{Discussion}

The coexistence of spontaneous intracranial dissections and saccular cerebral aneurysms is rare, but congenital or acquired fragility of the internal elastic lamina is likely to be the common contributing factor. Vascular dissection is thought to result from sudden longitudinal disruption of the internal elastic lamina..$^{9,10,17)}$ Saccular cerebral aneurysms are thought to originate in damage to the vascular endothelial cell layer and subsequent specific intimal changes such as fragmentation of the internal elastic lamina and thinning of the medial smooth muscle cell layer. ${ }^{11)} \mathrm{A}$ history of cigarette smoking, a known risk factor for saccular cerebral aneurysm, is often noted in patients with dissecting aneurysms. Interestingly, both of our patients had hypocholesterolemia including low apolipoprotein E, with total cholesterol levels of $98 \mathrm{mg} / \mathrm{dl}$ in Case 1 and $87 \mathrm{mg} / \mathrm{dl}$ in Case 2. Low apolipoprotein $\mathrm{E}$ level, a genetic factor in hypocholesterolemia, ${ }^{20)}$ is thought to be involved in the fragmentation of the internal elastic lamina, formation of aneurysms, and arterial wall dissection. ${ }^{2,8)}$ However, whether low apolipoprotein $\mathrm{E}$ levels increase the risk for formation of these two
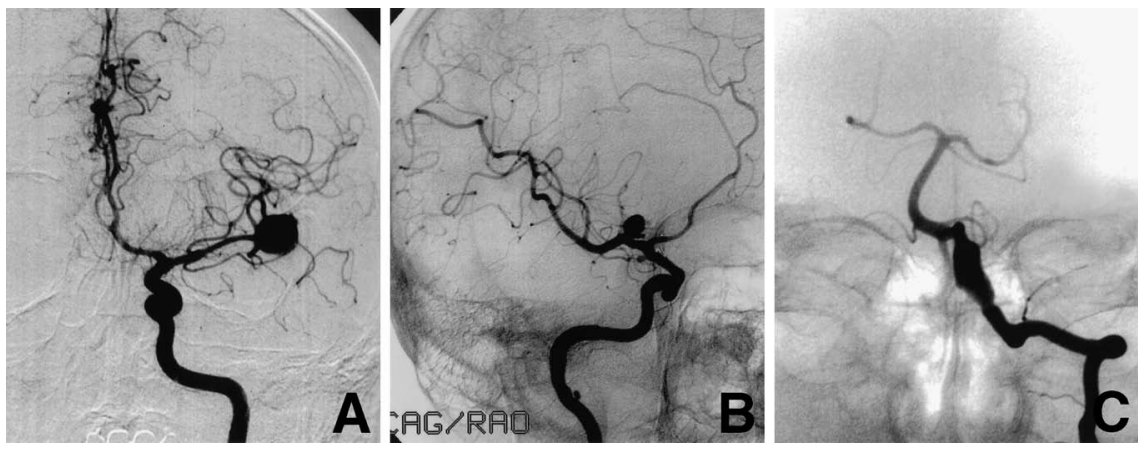

Fig. 5 Case 2. A: Left carotid angiogram, anteroposterior view, showing the largest aneurysm of the middle cerebral artery. B: Right carotid angiogram, right anterior oblique view, demonstrating aneurysms of the middle cerebral artery and anterior choroidal artery. A small pouch on the anteromedial wall of the extracranial carotid artery suggests vascular wall dissection. C: Left vertebral angiogram, anteroposterior view, showing a dissecting aneurysm of the left vertebral artery. 
distinct types of cerebral aneurysm remains unclear. Study of larger patient populations is required to identify the common risk factors that contribute to the simultaneous formation of saccular and dissecting cerebral aneurysms.

The clinical management of patients with $\mathrm{SAH}$ associated with both dissection and saccular aneurysms is complicated. The bleeding site should be very carefully identified in these patients. Preoperative identification of the rupture site relies on the angiographic depiction of the size and shape of multiple saccular aneurysms. The ruptured saccular aneurysm tends to be larger and irregular in shape. ${ }^{3)}$ The CT location of the hematoma, especially if coinciding with the site of the larger aneurysm, is a good indicator of the bleeding lesion. However, angiographic differentiation between ruptured and unruptured dissecting aneurysms is difficult, and some dissections are visualized as arterial stenosis or are angiographically occult. ${ }^{4,14,16)}$

The key for the therapeutic strategy of patients with multiple aneurysms is the decision regarding the management of associated unruptured lesions. There appears to be consensus on the surgical strategies to approach multiple saccular aneurysms. The identified associated saccular aneurysms should be secured in the same session, if possible. However, the choice of one-stage or two-stage clipping surgery depends on the severity of SAH, associated with brain swelling, and the sites of the aneurysms. ${ }^{3)}$ In general, contralateral associated aneurysms are not clipped in the presence of severe SAH. Endovascular treatment can safely obliterate all aneurysms in one session regardless of their location, even during the acute stage of severe SAH. ${ }^{19)}$ On the other hand, there is no consensus for the management of associated dissecting aneurysm. Asymptomatic dissecting aneurysms have a benign clinical course in general, ${ }^{7,21)}$ so can also be monitored during the acute stage of SAH. However, our Case 1 had new bleeding during the postoperative course, so we speculated that the hemodynamic stress related to the onset of $\mathrm{SAH}$ triggered the rupture.

Hemodynamic stress may be the cause of the asymptomatic dissection. Two of three cases of dissecting aneurysms of the bilateral vertebral arteries with SAH had new bleeding from the contralateral vertebral artery soon after treatment of the ruptured vessel. ${ }^{15)}$ A vertebral artery dissection demonstrating string sign developed secondary aneurysmal formation under the continuous hypertension. ${ }^{13)}$ In addition, the risk of bleeding from unruptured dissecting aneurysms of the vertebral artery is higher than previously considered, and patients with aneurysmal dilatation or typical pearl-and-string sign are at high risk. ${ }^{12,13)}$ Therefore, control of blood pressure during the acute stage of $\mathrm{SAH}$ is essential for such patients. However, this conservative treatment cannot be sustained in the presence of symptomatic cerebral vasospasm as in our Case 2.

Our present and previous experience suggest that prophylactic obliteration of the associated unruptured dissecting aneurysm in the acute stage of SAH, especially in the presence of evidence of aneurysmal dilatation or typical pearl-and-string sign, appears to provide better outcomes. Endovascular treatment can be considered as the first treatment, and endovascular trapping is safe for lesions not involving perforating arteries to the cerebellum or brain stem. ${ }^{5)}$ The treatment strategy of SAH patients with both dissecting and saccular type aneurysms is not clear. Further studies are required to establish the therapeutic strategy for patients presenting with $\mathrm{SAH}$ associated with both intracranial dissecting aneurysms and saccular cerebral aneurysm.

\section{References}

1) Anxionnat R, de Melo Neto JF, Bracard S, Lacour JC, Pinelli C, Civit T, Picard L: Treatment of hemorrhagic intracranial dissections. Neurosurgery 53: 289-301, 2003

2) Chen J, Kuhlencordt PJ, Astern J, Gyurko R, Huang PL: Hypertension does not account for the accelerated atherosclerosis and development of aneurysms in male apolipoprotein E/endothelial nitric oxide synthase double knockout mice. Circulation 104: 2391-2394, 2001

3) Hernesniemi J, Rinne J: Multiple aneurysms. Surg Neurol 60: 136-137, 2003

4) Hosoya T, Adachi M, Yamaguchi K, Haku T, Kayama T, Kato T: Clinical and neuroradiological features of intracranial vertebrobasilar artery dissection. Stroke 30: 1083-1090, 1999

5) Iihara K, Sakai N, Murao K, Sakai H, Higashi T, Kogure S, Takahashi JC, Nagata I: Dissecting aneurysms of the vertebral artery: a management strategy. J Neurosurg 97: 259-267, 2002

6) Ikeda T, Kurita H, Konishi Y, Fujitsuka M, Hino K, Shiokawa Y, Saito I: De novo dissecting aneurysm in a patient with a ruptured saccular lesion. J Neurosurg 97: 701-704, 2002

7) Kitanaka C, Tanaka J, Kuwahara M, Teraoka A, Sasaki T, Takakura K: Nonsurgical treatment of unruptured intracranial vertebral artery dissection with serial follow-up angiography. J Neurosurg 80: 667-674, 1994

8) Kuhlencordt PJ, Gyurko R, Han F, Scherrer-Crosbie $\mathrm{M}$, Aretz $\mathrm{TH}$, Hajjar R, Picard $\mathrm{MH}$, Huang $\mathrm{PL}$ : Accelerated atherosclerosis, aortic aneurysm formation, and ischemic heart disease in 
apolipoprotein E/endothelial nitric oxide synthase double-knockout mice. Circulation 104: 448-454, 2001

9) Mizutani T, Aruga T, Kirino T, Miki Y, Saito I, Tsuchida T: Recurrent subarachnoid hemorrhage from untreated ruptured vertebrobasilar dissecting aneurysms. Neurosurgery 36: 905-913, 1995

10) Mizutani T, Kojima H, Asamoto S, Miki Y: Pathological mechanism and three-dimensional structure of cerebral dissecting aneurysms. J Neurosurg 94: 712-717, 2001

11) Morimoto $M$, Miyamoto S, Mizoguchi A, Kume $N$, Kita T, Hashimoto $\mathrm{N}$ : Mouse model of cerebral aneurysm: experimental induction by renal hypertension and local hemodynamic changes. Stroke 33: 1911-1915, 2002

12) Naito I, Iwai T, Sasaki T: Management of intracranial vertebral artery dissections initially presenting without subarachnoid hemorrhage. Neurosurgery 51: 930-937, 2002

13) Nakagawa $K$, Touho $H$, Morisako $T$, Osaka $Y$, Tatsuzawa K, Nakae H, Owada K, Matsuda K, Karasawa J: Long-term follow-up study of unruptured vertebral artery dissection: clinical outcomes and serial angiographic findings. J Neurosurg 93: $19-25,2000$

14) Nohjoh T, Houkin K, Takahashi A, Abe H: Ruptured dissecting vertebral artery aneurysm detected by repeated angiography: case report. Neurosurgery 36: 180-182, 1995

15) Otawara Y, Ogasawara K, Ogawa A, Kogure T: Dissecting aneurysms of the bilateral arteries with subarachnoid hemorrhage: report of three cases.
Neurosurgery 50: 1372-1374, 2002

16) Rinkel GJ, van Gijn J, Wijdicks EF: Subarachnoid hemorrhage without detectable aneurysm. A review of the causes. Stroke 24: 1403-1491, 1993

17) Sakata N, Takebayashi S, Kojima M, Masawa N, Suzuki K, Takatama M: Pathology of a dissecting intracranial aneurysm. Neuropathology 1: 104-108, 2000

18) Sen J, Belli A, Albon H, Morgan L, Petzold A, Kitchen N: Triple-H therapy in the management of aneurysmal subarachnoid haemorrhage. Lancet Neurol 2: 614-621, 2003

19) Solander S, Ulhoa A, Vinuela F, Duckwiller GR, Gobin YP, Martin NA, Frazee JG, Guglielmi G: Endovascular treatment of multiple intracranial aneurysms by using Guglielmi detachable coils. J Neurosurg 90: 857-864, 1999

20) Synder SM, Terdiman JF, Caan B, Feingold KR, Hubl ST, Smith RS, Young SG: Relationship of apolipoprotein E phenotypes to hypocholesterolemia. Am J Med 95: 480-488, 1993

21) Yoshimoto Y, Wakai S: Unruptured intracranial vertebral artery dissection. Clinical course and serial radiographic imagings. Stroke 28: 370-374, 1997

Address reprint requests to: Yasuhiko Akiyama, M.D., Department of Neurosurgery, Shimane University School of Medicine, 89-1 Enya-cho, Izumo, Shimane 693-8501, Japan.

e-mail: yakiyama@med.shimane-u.ac.jp 